Información farmacológica

POLÍTICAS SOBRE MEDICAMENTOS

ENMIENDAS A LA ROTULACIÓN

USO RACIONAL

RETIROS DEL MERCADO

REACCIONES ADVERSAS

DECISIONES DIVERSAS
Propuesta de ley suiza sobre medicamentos; directrices sobre anuncios de medicamentos dirigidos directamente al consumidor por la radio y la televisión.

Enmiendas al rótulo del envase de paracetamol para advertir sobre el peligro de una sobredosis; cambios en la rotulación de la troglitazona debido al peligro de hepatotoxicidad; nuevas contraindicaciones en el envase del flumazenil; advertencias en el rótulo sobre el uso de la triamcinolona durante el embarazo.

Peligro de interacción entre la toronja y sus productos y algunos medicamentos; medidas para prevenir los errores de medicación; ubicación, tamaño y visibilidad del nombre de los medicamentos en los anuncios y en el rótulo comercial.

Mandamiento judicial del gobierno estadounidense contra quienes promueven productos no autorizados para tratar el cáncer y otras enfermedades; falsificación de antimaláricos; retiro del bromfenaco del mercado por hepatotoxicidad; retiro del dipiridamol debido a pruebas insuficientes de eficacia para el tratamiento de la angina pectoris; retiro de diversos medicamentos por entidad argentina; retiro del mercado de la vacuna RotaShield ${ }^{\circledR}$ contra rotavirus.

Trastornos digestivos asociados con el uso del alendronato; reacciones neurotóxicas mortales asociadas con el uso de un sellante quirúrgico a base de fibrina humana; notificación de reacciones adversas asociadas con el uso de antagonistas de los receptores de la angiotensina II; interacción entre los macrólidos y diversos medicamentos.

Aprobación del orlistat por la FDA para el tratamiento de la obesidad; aprobación por la FDA del uso del fosfato de oseltamivir en pacientes con síntomas tempranos de influenza.

\section{POLÍTICAS SOBRE MEDICAMENTOS}

\section{Ley sobre medicamentos: se presenta propuesta de ley al Parlamento (Suiza)}

Según ha declarado la Oficina Federal de Salud Pública, el Consejo Federal ha presentado ante el Parlamento una propuesta de ley donde se plantea la creación de un instituto para el control de los productos medicamentosos y se proponen posibles soluciones a algunos problemas, tales como la venta de medicamentos por correo (lo cual incluye su venta por la Internet) y la importación paralela. Hasta la fecha, estos productos han sido regulados por cada cantón y la Unión Intercantonal para el Control de los Medicamentos (IUCM) ha tratado de elaborar un acuerdo mediante el cual se buscaría una concordancia entre las regulaciones locales y la Ley Europea. La nueva propuesta de ley, que garantiza el registro de sustancias terapéuticas de calidad, inocuas y eficaces, está orientada a proteger la salud de los seres humanos y animales. En lo que respecta a los medicamentos, son varios los medios con los que se piensa cumplir este objetivo y entre ellos figuran un sistema que abarca la aprobación comercial, la vigilancia, el control de calidad y la consecución obligatoria de autorización por fabricantes y mayoristas.

En lo referente a los aparatos de tipo terapéutico, las medidas de control de calidad establecidas por el fabricante y el distribuidor tienen importancia prioritaria, ya que no es obligatorio conseguir una autorización para comercializar estos productos. La función de las autoridades reguladoras 
consistirá principalmente en monitorear la comercialización de estos aparatos y en cerciorarse de que satisfagan los requisitos establecidos para la protección de la salud y la prevención de actividades fraudulentas.

La venta de medicamentos por correo sigue siendo prohibida en principio a fin de garantizar la inocuidad de los productos y de proteger al paciente. De esta forma se busca evitar, por ejemplo, que los medicamentos cuya venta exige una receta médica se vendan por la Internet sin presentar dicha receta o sin ningún tipo de control. No obstante, la venta por correo de medicamentos prescritos por un médico se permitirá siempre y cuando se satisfagan los requisitos de seguridad.

\section{Directrices finales de la FDA sobre los anuncios dirigidos directamente al consumidor para la venta de medicamentos que solo se consiguen con receta médica (Estados Unidos)}

La Administración de Alimentos y Medicamentos (FDA) ha emitido sus directrices finales sobre los anuncios de radio o televisión que informan directamente al consumidor sobre la venta de medicamentos obtenibles solamente con receta médica. Estas directrices serán continuación de una estrategia polifacética para lograr que los productos anunciados por la radio o la televisión sean debidamente identificados. De ordinario se dan las cuatro fuentes siguientes: 1) un número telefónico gratuito; 2) la remisión a un anuncio seriado publicado simultáneamente en una fuente impresa, o la provisión de suficientes folletos en diversos puntos de fácil acceso; 3) la remisión a un proveedor de servicios de salud (sea médico, farmacéutico, veterinario $\mathrm{u}$ otro); o 4) una página en la Internet.

Tal como lo exigen los reglamentos aplicables a la comercialización de medicamentos obtenibles con receta médica, los anuncios emitidos por la radio, la televisión o las vías telefónicas deben consistir en una "declaración extensa" donde se expongan de manera clara todos los riesgos importantes que se asocian con el medicamento.

Las directrices finales han sufrido algunas enmiendas menores:

- Se ha eliminado la opción de proporcionar a los consumidores el rótulo del producto si llaman al número telefónico gratuito.

- Se ha hecho hincapié en la importancia de diseminar el componente impreso de la estrategia que busca garantizar la provisión adecuada del producto.

- Se ha reconocido que el componente de la estrategia antedicha que consiste en proporcionar folletos se podría poner en práctica únicamente cuando fueran escasos los anuncios por la radio o la televisión.

- Se ha reconocido abiertamente que los proveedores de atención de salud que no son médicos o farmacéuticos pueden ser fuentes de información adicional sobre productos medicamentosos para consumo humano.

- Se han aclarado las diferencias estratégicas que entrañan los anuncios emitidos por el teléfono, la televisión o la radio.

\section{ENMIENDAS A LA ROTULACIÓN}

\section{Se enmienda la rotulación del paracetamol: advertencia sobre el peligro de una sobredosis (Suecia)}

La Agencia de Productos Medicamentosos actualmente exige que se incorporen nuevas advertencias en la rotulación del paracetamol debido a que una dosis excesiva puede provocar graves reacciones adversas, entre ellas trastornos hepáticos.

La siguiente declaración debe colocarse en todas las fuentes de información sobre el producto, en los prospectos que se incluyen en el envase y en el rótulo.

¡Advertencia! La ingestión de dosis mayores de las recomendadas puede conducir a un riesgo de sufrir trastornos hepáticos graves. No use este producto sin receta médica si sufre de alcoholismo o de alguna enfermedad hepática, o si consume otros productos a base de paracetamol.

La colocación de esta nueva advertencia en la rotulación del producto ha sido obligatoria a partir del 1 de abril de 1999.

\section{Se enmiendan la rotulación y las indicaciones de Rezulin ${ }^{\circledR}$ (Estados Unidos)}

Se han hecho enmiendas importantes a la rotulación y a las indicaciones para el uso de Rezulin ${ }^{\circledR}$ (troglitazona), medicamento que se usa para el tratamiento de la diabetes mellitus tipo 2 (diabetes sin dependencia a la insulina o diabetes del adulto). Estas enmiendas obedecen a que hay datos nuevos (es decir, pruebas adicionales de que puede producirse daño hepático grave $y$ en ocasiones mortal en pacientes tratados con Rezulin ${ }^{\circledR}$ ) según los cuales el uso de Rezulin ${ }^{\circledR}$ debe limitarse a pacientes cuya enfermedad no puede controlarse con otros tratamientos y no debe usarse como tratamiento único inicial para la diabetes tipo 2. Entre las enmiendas a la rotulación también figura la recomendación de vigilar más de cerca la función hepática en pacientes que consumen Rezulin ${ }^{\circledR}$.

Parke-Davis ha emitido una carta a los profesionales de la salud de todo el país para notificarles de estas modificaciones. La carta dice así:

- Rezulin ${ }^{\circledR}$ ya no está indicado como medicamento único inicial.

- Los pacientes que serán tratados con Rezulin ${ }^{\circledR}$ tienen que ser sometidos a pruebas de función hepática antes de iniciar el tratamiento, y posteriormente cada mes durante el primer año (y no 10 veces únicamente, como se solía recomendar). Después del primer año de tratamiento, los pacientes deben ser sometidos a estas pruebas cada trimestre (y no "periódicamente", como se pedía antes).

- La compañía ha preparado una hoja de información para el paciente con los datos proporcionados por la Administración de Alimentos y Medi- 
camentos (FDA) sobre el uso inocuo y eficaz de Rezulin ${ }^{\circledR}$. Esta hoja es distribuida por los farmacéuticos cada vez que un paciente presenta una receta por Rezulin ${ }^{\circledR}$.

\section{Nuevas contraindicaciones para el uso del flumazenil (Reino Unido)}

Roche, compañía que fabrica el antagonista de las benzodiazepinas denominado flumazenil (Anexate ${ }^{\circledR}$ ), ha agregado al prospecto del medicamento algunos datos nuevos sobre sus características farmacocinéticas. Las secciones correspondientes a su distribución, metabolismo, eliminación y rasgos farmacocinéticos en situaciones especiales han sido enmendadas.

El prospecto también contiene ahora una advertencia sobre el uso contraindicado del flumazenil en pacientes que han sido tratados con una benzodiazepina para el control de un problema de salud potencialmente mortal (e.g., el control de la presión intracraneana o de un estado epiléptico). En la sección de precauciones, el prospecto actual indica que el uso del flumazenil no se recomienda en pacientes epilépticos que han recibido un tratamiento prolongado con benzodiazepinas y que el medicamento debe administrarse con cautela a pacientes que han sufrido un traumatismo craneano. También se explica ahora en esta sección que cuando se administra el flumazenil en combinación con bloqueadores neuromusculares, no se debe inyectar hasta que hayan desaparecido por completo los efectos del bloqueo neuromuscular; añade que el medicamento no es un tratamiento recomendado para la dependencia de las benzodiazepinas o para los síndromes de abstinencia que perduran largo tiempo tras la suspensión de estos fármacos.

\section{Nuevas especificaciones sobre el uso del hexacetónido de triamcinolona durante el embarazo (Reino Unido)}

Para responder a una solicitud de la Agencia para el Control de los Medica- mentos, el fabricante del hexacetónido de triamcinolona (Lederspan ${ }^{\circledR}$ : Wyeth) ha revisado el prospecto sobre el medicamento. La sección sobre el embarazo y la lactancia ha sido actualizada y contiene información sobre el paso transplacentario de la triamcinolona. Dice así el prospecto actual:

- Puede haber un mayor riesgo de retraso del crecimiento intrauterino si se administra un tratamiento largo con corticoesteroides y hay, en teoría, un peligro de hipoadrenalismo en el neonato.

- Cuando es esencial la administración de corticoesteroides, las pacientes con embarazos normales pueden ser tratadas como si no estuvieran embarazadas.

- Los corticoesteroides pueden llegar a la lecha materna, aunque no hay datos disponibles en el caso de la triamcinolona.

- Los lactantes cuyas madres consumen altas dosis de corticoesteroides sistémicos por un período prolongado pueden tener cierto grado de supresión suprarrenal.

La sección sobre reacciones adversas también ha sido revisada y ahora contiene información sobre la calcinosis.

\section{USO RACIONAL}

\section{La advertencia sobre la toronja adquiere carácter oficial en algunos países (Nueva Zelandia)}

"No ingiera toronjas o su jugo mientras esté en tratamiento con este medicamento", dicen literalmente los rótulos que ponen al paciente sobre alerta con respecto al peligro de posibles interacciones entre la toronja y el fármaco. Esta nueva advertencia se ha creado después de descubrir que la toronja puede aumentar notablemente las concentraciones plasmáticas de varios medicamentos.

Los medicamentos enumerados a continuación deben llevar una advertencia sobre su posible interacción con la toronja:
- Benzodiazepinas (alprazolam, midazolam, triazolam)

- Antagonistas del calcio

- Cafeína

- Cisaprida

- Clomipramina

- Ciclosporina

- Etiniloestradiol

- Inhibidores de la reductasa de la 3-hidroxil-3-metilglutaril (HMG)coenzima A

- Antihistamínicos sin efecto sedante

- Quinidina

- Tacrolimús

La mayor parte de estos fármacos tienen en común que sufren un primer metabolismo intenso por parte de la citocromoxidasa P450 (enzima CYP3A4/5). La inhibición de esta enzima por la toronja puede llevar a un aumento de las concentraciones plasmáticas del medicamento y a interacciones clínicas importantes.

Actualmente, la toronja se destaca entre las frutas y legumbres por su grado de interferencia con los medicamentos. Por añadidura, la cantidad de jugo de toronja que se necesita es relativamente pequeña; un vaso de jugo de $250 \mathrm{~mL}$ puede inhibir notablemente la CYP3A4. El efecto de la toronja en el metabolismo del fármaco perdura 24 horas después de la ingestión de un solo vaso de jugo.

La toronja, curiosamente, puede usarse para reducir la cantidad del medicamento. De hecho, algunos pacientes han logrado reducir la dosis de ciclosporina mediante su ingestión. No obstante, sería quizá más racional crear una molécula con un sabor amargo similar al de esta fruta.

\section{La prevención de los errores de medicación (Estados Unidos)}

El Institute for Safe Medication Practices (ISMP) de los Estados Unidos recomienda algunas estrategias para prevenir los errores de medicación. Estas se enumeran en el orden de su capacidad para engendrar cambios perdurables conducentes al uso seguro de los medicamentos. 
Medidas destinadas a reducir el mal uso de los medicamentos:

- Quitar los preparados concentrados de cloruro de potasio inyectable de todas las zonas donde se trate a los pacientes

- Para administrar por la vía oral cualquier medicamento líquido, usar vasitos o jeringas especiales para uso oral (no jeringas parenterales) que no puedan conectarse a los tubos de las soluciones endovenosas

- Impedir que el personal de enfermería tenga acceso a la farmacia cuando esté cerrada mediante la creación de un gabinete o dispensario nocturno debidamente surtido

La automatización y el uso de computadoras:

La tecnología moderna aplicada a las actividades y procesos involucrados en el uso de los medicamentos pueden aminorar la falibilidad humana porque limitan la dependencia de la memoria. Como ejemplos pueden citarse el uso de sistemas de información farmacológica computadorizados que tengan la debida integridad tecnológica y clínica; el pedido directo del medicamento por el propio médico, durante el cual este tiene acceso a información y advertencias sobre el producto; y el uso de bombas de infusión endovenosa con mecanismos a toda prueba para así evitar el flujo libre de la solución.

Protocolos y formularios estandarizados:

Estos ofrecen una guía para el uso seguro de los medicamentos, puesto que eliminan los problemas de ilegibilidad y logran que se establezca una forma de comunicación segura para hacer los pedidos. No obstante, como medidas para la prevención de errores suelen ser menos confiables que las mencionadas anteriormente, puesto que su observación depende de la vigilancia humana. En ocasiones, sin embargo, podrían constituir la medida más apropiada, o quizá la única, para remediar un problema relacionado con el uso de un medicamento.
La verificación:

Los sistemas de verificación independientes y otras medidas redundantes, en las que un individuo revisa el trabajo de otro, son herramientas que pueden reducir el riesgo de cometer errores. Las posibilidades de que dos individuos cometan el mismo error al administrar el mismo medicamento al mismo paciente son sumamente remotas. No obstante, la posibilidad de una equivocación sigue existiendo, ya que esta estrategia se orienta más hacia la detección que hacia la prevención de errores.

\section{Reglamentos y políticas:}

Mediante esta estrategia se busca controlar el comportamiento humano, no necesariamente arreglar el sistema. Aunque es útil y necesaria, debe aplicarse principalmente como un respaldo a otras medidas más eficaces para la prevención de errores que sí estén diseñadas para mejorar el sistema.

\section{Educación e información:}

La educación del personal puede ser importante si se combina con otras estrategias que refuercen el control del uso de los medicamentos. No obstante, a menudo se pasa por alto la continuidad que deben tener los programas educativos eficaces y se olvida lo poco realista que es su dependencia de un rendimiento humano libre de errores.

\section{Ubicación, tamaño y visibilidad del nombre del medicamento en los anuncios (Estados Unidos)}

La Administración de Alimentos y Medicamentos (FDA) ha anunciado la disponibilidad de un documento preliminar de orientación titulado "Product Name Placement, Size, and Prominence in Advertising and Promotional Labeling" [Ubicación, tamaño y visibilidad del nombre del producto en los anuncios y rótulos comerciales]. Al documento se le han añadido nuevas secciones donde se examina su aplicabilidad a los anuncios audiovisuales, a los que salen en la radio, la televisión o la computadora, y a los rótulos comerciales; también se le ha agregado una sección sobre la ubicación, el tamaño y la visibilidad del nombre comercial y del nombre genérico de los productos que contienen dos o más ingredientes activos. En el documento se examina la aplicabilidad de las orientaciones a los medicamentos de origen animal que se adquieren con receta médica y a los productos biológicos.

\section{RETIROS DEL MERCADO}

\section{Medicamentos no autorizados para el tratamiento del cáncer (Estados Unidos)}

La Administración de Alimentos y Medicamentos (FDA) ha solicitado un mandamiento judicial en contra de la comercialización de tres medicamentos no autorizados que se están promoviendo ilegalmente como tratamientos contra el cáncer y otras enfermedades por conducto de la corporación Lane Labs-USA, Inc., situada en Allendale, Nueva Jersey, y su presidente. Los productos en cuestión son los siguientes:

- "BeneFin", elaborado con cartílago de tiburón y promovido como tratamiento contra el cáncer y otras enfermedades

- "SkinAnswer", pomada a base de glucoalcaloides que se vende como tratamiento contra el cáncer de la piel

- "MGN-3", extracto de arroz y salvado que se recomienda como tratamiento contra el cáncer y la infección por el virus de la inmunodeficiencia humana (VIH), agente causal del sida

Se están llevando a cabo estudios con el "BeneFin" para determinar su posible utilidad contra el cáncer, siguiendo las pautas de la FDA para Medicamentos Experimentales Nuevos. Esto permite que se distribuya para uso en ensayos clínicos, pero como en el caso de otros productos, no puede promoverse o comercializarse sin ha- 
berse antes demostrado su inocuidad y eficacia y sin que la FDA haya revisado y aprobado las condiciones de venta establecidas por el patrocinador.

El mandamiento en contra del cartílago de tiburón de marca "BeneFin" no afecta a otras marcas del producto que no se destinan al tratamiento de enfermedades y cuya venta como suplementos dietéticos es legal.

En junio de 1997 la FDA advirtió a los acusados que sus actividades de promoción eran ilegales, pero estos siguen promoviendo el "BeneFin", "SkinAnswer" y "MGN-3" como remedios contra el cáncer y otras enfermedades, valiéndose para ello de diversos medios, como libros, artículos, sitios en la Internet y las declaraciones de sus empleados. La solicitud de un mandamiento judicial permanente por parte del gobierno se basa en que los acusados no se muestran dispuestos a obedecer la ley. El gobierno busca, entre otras cosas, que se prohíba a los acusados seguir distribuyendo los tres medicamentos no autorizados, a no ser que la FDA apruebe su comercialización o permita su distribución bajo la disposición sobre Medicamentos Experimentales Nuevos solamente para fines de la conducción de ensayos clínicos.

\section{Dos medicamentos antimaláricos fraudulentos (Camboya)}

El artesunato es una versión falsificada del producto elaborado en la China por Guilin Pharmaceutical Factory. En un comienzo este se vendía en paquetes que carecían del holograma característico que sí aparece en el producto legítimo. Sin embargo, recientemente ha habido informes al efecto de que los medicamentos fraudulentos ahora vienen en envases con un "holograma falsificado" en la película de aluminio que recubre el blíster. El "holograma falsificado" muestra la misma vista del lago Guilin rodeada de un círculo, con un diámetro aproximado de $0,5 \mathrm{~m}$, que se encuentra en el aluminio del envase original, pero no cambia de color cuando se inclina.

En Camboya hay una amplia distribución de botellas de 100 tabletas cuyo rótulo dice "mefloquina" y "Gateway Pharmaceuticals". Estas botellas, que no contienen mefloquina, se venden a un precio de US $\$ 7,00$ por 100 tabletas, mucho menos de lo que cuesta la mefloquina genuina más barata en todo el mercado mundial. Gateway Pharmaceuticals de Australia vende mefloquina, pero en botellas de 25 tabletas que se rotulan "Meflam". A diferencia de las tabletas fraudulentas, cada tableta de la mefloquina genuina que vende la compañía Gateway lleva impresa la marca $G / W$.

\section{Retiro del bromfenaco por hepatotoxicidad (Arabia Saudita)}

En consonancia con las medidas dictaminadas por la Administración de Alimentos y Medicamentos (FDA) de los Estados Unidos de América, el Ministerio de Salud ha retirado del mercado todos los productos a base de bromfenaco (Duract ${ }^{\circledR}$ : Wyeth Ayerst) porque ha habido informes de insuficiencia hepática, que en ocasiones ha sido mortal.

\section{Retiro del dipiridamol por ser insuficientes las pruebas de su eficacia (Estados Unidos)}

La Administración de Alimentos y Medicamentos (FDA) ha retirado su autorización condicional de ciertos productos a base de dipiridamol porque no hay suficientes datos que confirmen su eficacia como tratamiento prolongado de la angina pectoris crónica.

Se había concedido la autorización bajo la condición de que se proveyeran pruebas adicionales de que el producto es eficaz para este trastorno. Dado que dichas pruebas no se han proporcionado, la agencia ha retirado su aprobación del producto para esta indicación. Se han autorizado productos a base de dipiridamol cuando se usan como "coadyuvantes de los anticoagulantes cumarínicos para prevenir las complicaciones tromboembólicas tras el reemplazo de válvulas cardíacas".

\section{Retiros del mercado por la Administración Nacional de Medicamentos, Alimentos y Tecnología Médica (Argentina)}

Según la disposición 5740/99 del 14 de octubre de 1999 y en el marco del "Programa de Pesquisa de Medicamentos Ilegítimos", agentes del Instituto Nacional de Medicina (INAME) realizaron en distintos puntos de la Capital Federal, de la provincia de Buenos Aires y de la provincia de Mendoza una serie de procedimientos que culminaron en el retiro de unidades de los siguientes productos:

- Sertal Compuesto ${ }^{\circledR}$, marca registrada de la firma Roemmers S.A.I.C.F., en envases de 50 comprimidos

- Dorixina ${ }^{\circledR}$, marca registrada de la firma Roemmers S.A.I.C.F., en envases de 50 comprimidos

- Novalgina ${ }^{\circledR}$, marca registrada de la firma Hoechst Marion Roussel Argentina, S.A., en envases de 50 comprimidos

- Bayaspirina ${ }^{\circledR}$, producto de la compañía Bayer Argentina, S.A., en envases de 100 comprimidos

- Migral $500^{\circledR}$, marca registrada de la firma Química Montpellier, S.A., en envases de 50 comprimidos

- Bacticort Complex ${ }^{\circledR}$, marca registrada de la firma Química Montpellier, S.A., en envases de crema de $10 \mathrm{~g}$

- $\operatorname{Dolex}^{\circledR}$, marca registrada de la firma Syncro S.A.Q.I.C.I.F., en envases de 10 comprimidos

- Buscapina Compositum $\mathrm{N}^{\circledR}$, marca registrada de la firma Boerhinger Ingelheim, S.A., en envases de 50 comprimidos

- Ibuevanol ${ }^{\circledR}$, marca registrada de la firma Smithkline Beecham, en envases de 20 comprimidos

\section{Retiro de la vacuna RotaShield ${ }^{\circledR}$ contra rotavirus (Estados Unidos)}

Wyeth-Lederle, compañía estadounidense que fabrica la vacuna contra rotavirus denominada RotaShield ${ }^{\circledR}$, ha mandado una carta a los proveedores de atención de salud para avisarles 
que ha decidido retirar del mercado esta vacuna. La medida fue adoptada en consulta con la Administración de Alimentos y Medicamentos (FDA) después de que los Centros para el Control y la Prevención de Enfermedades (CDC) de Atlanta, Georgia, recomendaron posponer la administración del producto debido a la llegada de algunos informes al Sistema de Notificación de Reacciones Adversas a Vacunas (Vaccine Adverse Events Reporting System, VAERS) sobre una posible asociación entre el uso de RotaShield $^{\circledR}$ y episodios de intususcepción. Wyeth Lederle Vaccines, con ánimo de proteger los intereses del público, ha optado por retirar todos los lotes restantes de la vacuna.

\section{REACCIONES ADVERSAS}

\section{Efectos adversos asociados con el uso del alendronato (Australia)}

El alendronato sódico (Fosamax ${ }^{\circledR}$ ) se empezó a vender en Australia a fines de 1996 y desde entonces el Australian Drug Reactions Advisory Committee (ADRAC), en su informe publicado en agosto de 1999, señaló haber recibido 331 informes de reacciones adversas sospechadas y en $91 \%$ de los casos el alendronato fue el único medicamento en duda. Cincuenta y cuatro por ciento de los informes se relacionan con trastornos digestivos; el otro efecto importante se ha observado en el sistema musculoesquelético, que se mencionó en $18 \%$ de los informes.

Los efectos digestivos más destacados, que son la esofagitis y la úlcera esofágica, fueron notificados poco tiempo después de la comercialización del producto. Actualmente el ADRAC ha recibido 52 informes de esofagitis, ulceración esofágica o estenosis del esófago. También se han notificado al ADRAC casos de dispepsia (44), náusea (43), dolor abdominal (37) y disfagia (23). En los 180 informes de trastornos digestivos, las edades de los pacientes oscilaron entre 18 y 91 años (mediana: 71) y todos, a excepción de cinco, tenían como mínimo 50 años.
Ochenta y siete por ciento de los pacientes han sido de sexo femenino y la mayoría de los trastornos se han asociado con dosis diarias de $10 \mathrm{mg}$, aunque el resto de los pacientes consumían dosis de $40 \mathrm{mg}$.

También se han notificado 61 episodios de problemas musculoesqueléticos: 35 casos de dolor muscular, 29 casos de dolor articular y 6 casos de dolor óseo.

\section{Reacciones neurotóxicas mortales en conexión con el uso del sellador quirúrgico Quixil $^{\circledR}$ (Reino Unido)}

La Agencia para el Control de los Medicamentos ha emitido una advertencia a los profesionales de la salud sobre algunas dudas en conexión con la inocuidad de Quixil ${ }^{\circledR}$, producto sellador a base de fibrina plasmática humana y destinado al uso en cirugía para facilitar la hemostasis y reducir la hemorragia durante las operaciones de hígado. Contiene proteína coagulante y trombina humanas, así como diversos excipientes, entre ellos la glicina, la arginina y el ácido tranexámico. El producto está autorizado solamente en el Reino Unido, Israel, Brasil y México.

Desde que la venta del producto se autorizó en el Reino Unido, la Agencia ha recibido informes de reacciones neurotóxicas mortales en conexión con su uso no autorizado en procedimientos neuroquirúrgicos. Aunque estas podrían haber sido provocadas por varios de los constituyentes del producto, el mecanismo exacto de la reacción aún no se conoce. Como resultado de esta información, la Agencia ha emitido el siguiente mensaje de urgencia para todos los profesionales de la salud:

Quixil ${ }^{\circledR}$ no debe usarse en procedimientos quirúrgicos en los que se entra en contacto con el líquido cefalorraquídeo o la duramadre, como sería el caso de las operaciones neurológicas y de la espinal dorsal. Se reitera que Quixil ${ }^{\circledR}$ solo se autoriza para la cirugía del hígado y se advierte categóricamente que no debe prescribirse para otros propósitos.
En particular, no hay pruebas de inocuidad en procedimientos quirúrgicos cardiotorácicos, vasculares, neurológicos o de oídos, nariz y garganta.

Repaso de las reacciones adversas asociadas con los antagonistas de los receptores de la angiotensina II (Australia)

El losartán, sal potásica que se vende desde fines de 1997 con el nombre de Cozaar $^{\circledR}$, fue el primer antagonista de los receptores de la angiotensina II (ARA) en ser autorizado para el tratamiento de la hipertensión en Australia. Se comercializó después el irbesartán (Avapro ${ }^{\circledR}$, Karvea ${ }^{\circledR}$ ) a mediados de 1998 y se espera que en un futuro se vendan más productos de este tipo.

Hasta noviembre de 1998, el Australian Drug Reactions Advisory Committee (ADRAC) había recibido 230 informes de posibles reacciones adversas asociadas con el uso del losartán, más 133 informes similares en conexión con el uso del irbesartán. En la mayoría de los informes (64\%), el ARA era el único medicamento sospechado. La edad de los pacientes varió de 25 a 93 años (mediana: 67) y $68 \%$ eran mayores de 60 . Las reacciones casi siempre empezaron en las primera semanas del tratamiento y en 24 informes se observó una recurrencia de los síntomas cuando se volvió a administrar el fármaco.

\section{Principales interacciones medicamentosas asociadas con los macrólidos (Francia)}

Algunos macrólidos se asocian con un riesgo importante de interacciones medicamentosas peligrosas. Estas se indican en el cuadro 1.

\section{DECISIONES DIVERSAS}

\section{Autorización del orlistat para el tratamiento de la obesidad (Estados Unidos)}

La Administración de Alimentos y Medicamentos ha autorizado el uso de 
CUADRO 1. Principales interacciones medicamentosas de los macrólidos ${ }^{a}$

\begin{tabular}{|c|c|c|c|c|c|c|c|c|}
\hline & $\begin{array}{c}\text { Anticoagulante } \\
\text { oral }\end{array}$ & Astemizol & Bromocriptina & Carbamazepina & Cisaprida & Digoxina & Ergotamina & Teofilina \\
\hline Azitromicina & $++(b)$ & & (D) (c) & & (Cl) & $+($ d) & (Cl) & \\
\hline $\begin{array}{l}\text { Claritromicina } \\
\text { Diritromicina }\end{array}$ & ++ & (D) & $\begin{array}{l}++ \\
\text { (D) }\end{array}$ & $\begin{array}{c}+++ \\
(\mathrm{P})\end{array}$ & $\begin{array}{l}+++ \\
(\mathrm{Cl}) \\
(\mathrm{Cl})\end{array}$ & $\begin{array}{l}++ \\
(\mathrm{P})\end{array}$ & $\begin{array}{l}+ \\
(\mathrm{Cl}) \\
(\mathrm{Cl})\end{array}$ & $(\mathrm{TP})$ \\
\hline Eritromicina & $\begin{array}{l}+++ \\
(\mathrm{P})\end{array}$ & $\begin{array}{l}+++ \\
(\mathrm{Cl})\end{array}$ & $\begin{array}{l}++ \\
\text { (D) }\end{array}$ & $\begin{array}{l}+++ \\
\text { (D) }\end{array}$ & $\begin{array}{l}+++ \\
(\mathrm{Cl})\end{array}$ & $\begin{array}{c}+++ \\
(P)\end{array}$ & $\begin{array}{l}+++ \\
(\mathrm{Cl})\end{array}$ & $\begin{array}{c}+++ \\
\text { (D) }\end{array}$ \\
\hline Midecamicina & & (Cl) & (D) & + & (Cl) & & $\stackrel{+}{+}$ & \\
\hline $\begin{array}{l}\text { Roxitromicina } \\
\text { Espiramicina }\end{array}$ & ++ & & $\begin{array}{l}++ \\
\text { (D) }\end{array}$ & & (Cl) & + & (Cl) & $\begin{array}{c}+ \\
(\mathrm{TP})\end{array}$ \\
\hline
\end{tabular}

orlistat $\left(\right.$ Xenical $\left.^{\circledR}\right)$, medicamento nuevo fabricado por Roche Laboratories para el tratamiento de la obesidad. El orlistat es el primero de un nuevo grupo de fármacos contra la obesidad que se denominan inhibidores de las lipasas y que carecen de actividad sistémica. A diferencia de otros medicamentos para la obesidad, el orlistat impide que las enzimas del aparato digestivo reduzca los lípidos provenientes de los alimentos a moléculas más pequeñas que puedan ser absorbidas por el organismo. La absorción de lípidos se reduce en alrededor de $30 \%$. Como los triglicéridos que no se digieren no son absorbidos, la reducción de la ingesta calórica podría tener un efecto beneficioso en el control del peso.

Los efectos del orlistat en la pérdida, el mantenimiento y la recuperación del peso corporal y en diversas enfermedades relacionadas con la obesidad fueron evaluados en siete ensayos clínicos multicéntricos de larga duración. Estos estudios se realizaron con alrededor de 2800 pacientes tratados con orlistat y con 1400 pacientes que recibieron placebo. Se recomendó una dieta equilibrada y baja en calorías a todos los pacientes durante las fases de pérdida de peso y de mantenimiento. El propósito de la dieta fue re- ducir la ingestión de calorías en 20\% y lograr que los lípidos fueran la fuente de $30 \%$ de las calorías. Se dio orientación alimentaria a todos los pacientes.

De todos los pacientes que completaron un año de tratamiento, $57 \%$ de los tratados con orlistat y $31 \%$ de los que recibieron placebo perdieron un mínimo de $5 \%$ de su peso corporal inicial.

La dosis recomendada es una cápsula de orlistat con cada una de las comidas principales en las que se ingieran alimentos grasos. Durante el tratamiento, el paciente debe observar una dieta equilibrada y baja en calorías, 30\% de las cuales deben ingerirse en forma de lípidos. El orlistat está indicado para pacientes obesos con un índice de masa corporal (IMC, que es una medida del peso en relación con la estatura, según la fórmula peso $(\mathrm{kg})$ / estatura $\left(\mathrm{m}^{2}\right)$ ) de 30 o más, o para pacientes con un IMC de 27 o más que también son hipertensos, hipercolesterolémicos o diabéticos. Una persona que mide $5^{\prime} 5^{\prime \prime}(1,65 \mathrm{~m})$ de estatura y que pesa 180 libras $(81,6 \mathrm{~kg})$ tendría un IMC de 30.

Debido a que el orlistat reduce la absorción de algunas vitaminas liposolubles y de los carotenos beta, los pacientes deben tomar un suplemento que contenga vitaminas $\mathrm{A}, \mathrm{D}$ y $\mathrm{K}$ y dichos carotenos. Los efectos secundarios más comunes del orlistat son el manchado de la ropa interior por materia grasa, la flatulencia con escape fecal, la urgencia para defecar, la consistencia grasa de las heces y la defecación frecuente.

\section{Autorización de Tamiflu ${ }^{\circledR}$ para tratar los síntomas tempranos de la influenza (Estados Unidos)}

La Administración de Alimentos y Medicamentos (FDA) ha autorizado el uso de Tamiflu ${ }^{\circledR}$ (fosfato de oseltamivir), fármaco antirretrovírico que se administra por la vía oral, para tratar la influenza sin complicaciones en adultos cuyos síntomas no han durado más de dos días. Este producto se autoriza para el tratamiento de las influenzas de tipo A y B, aunque la mayoría de los pacientes que participaron en los estudios tenían el tipo A.

Se efectuaron dos ensayos clínicos controlados en los que participaron más de 100 pacientes. Uno de ellos tuvo lugar en los Estados Unidos y en el otro los pacientes provinieron de Canadá, Europa y Hong Kong. En estos estudios, el tratamiento con Ta- 
miflu $^{\circledR}$ se inició no más de 40 horas después de la aparición de los primeros síntomas. A los sujetos estudiados se les pidió que hicieran una autoevaluación de sus síntomas gripales. Se calculó el tiempo transcurrido desde el inicio del tratamiento hasta que todos los síntomas (congestión nasal, tos, dolor de garganta, malestar general, cansancio, cefaleas y sudación con escalofríos) disminuyeron hasta casi desaparecer, según el criterio de los pacientes. En ambos estudios, la reducción del tiempo transcurrido hasta el alivio de los síntomas fue de un poco más de un día en pacientes infectados que recibieron Tamiflu ${ }^{\circledR}$, cuando este grupo se comparó con el que recibió placebo.

Los efectos secundarios de Tamiflu ${ }^{\circledR}$ que se notificaron con mayor frecuencia fueron náusea, vómitos, insomnio y vértigo. No se ha demostrado que el medicamento sea eficaz para prevenir la influenza o para reducir el riesgo de transmitir el virus a otras personas. No está autorizado para uso en niños menores de 18 años. La eficacia de Tamiflu $^{\circledR}$ en sujetos con enfermedades cardíacas o respiratorias de carácter crónico aún no se ha determinado.
La dosis recomendada de Tamiflu ${ }^{\circledR}$ es de una cápsula de $75 \mathrm{mg}$ dos veces al día durante 5 días. Puede tomarse con o sin alimentos.

\section{REFERENCIAS}

Australian Adverse Drug Reaction Bulletin. Vol. 18, No. 3, agosto de 1999.

Food and Drug Administration. Talk Paper. 26 de abril de 1999.

Food and Drug Administration. Talk Paper. 16 de junio de 1999.

Food and Drug Administration. Talk Paper. 6 de agosto de 1999.

Food and Drug Administration. Talk Paper. 27 de octubre de 1999.

Food and Drug Administration. Talk Paper. 10 de diciembre de 1999.

Medifile Drug Information Bulletin. Vol. 13, No. 08, agosto de 1999.

Prescrire International. Vol. 8, No. 44, diciembre de 1999.

WHO DRS Information Exchange System. Alert. No. 89-93.

WHO/EDU. Memorándum 24649. 17 de diciembre de 1999 .
WHO Pharmaceuticals Newsletter. No. 5/6, mayo y junio de 1999.

www.anmat.gov.ar/prensa 44.html.

Información farmacológica da a conocer las decisiones oficiales sobre regulación de productos farmacéuticos adoptadas por organismos gubernamentales e internacionales en todo el mundo, los fundamentos científicos en que se sustentan tales decisiones y otros datos de interés relacionados con el tema. Como la mayor parte de la información proviene de fuentes de circulación relativamente limitada, su diseminación en esta forma permite hacerla llegar a un público más amplio. De esta manera se pretende contribuir a limitar el uso irracional de medicamentos y fomentar su uso racional en la Región de las Américas. La sección está a cargo del Programa de Medicamentos Esenciales y Tecnología para la Salud (HSE) de la OPS y se publica en la Revista Panamericana de Salud Públical Pan American Journal of Public Health en enero, abril, julio y octubre. Las separatas pueden solicitarse al programa mencionado, Organización Panamericana de la Salud, 525 Twenty-third Street, NW, Washington, DC 20037, EUA.

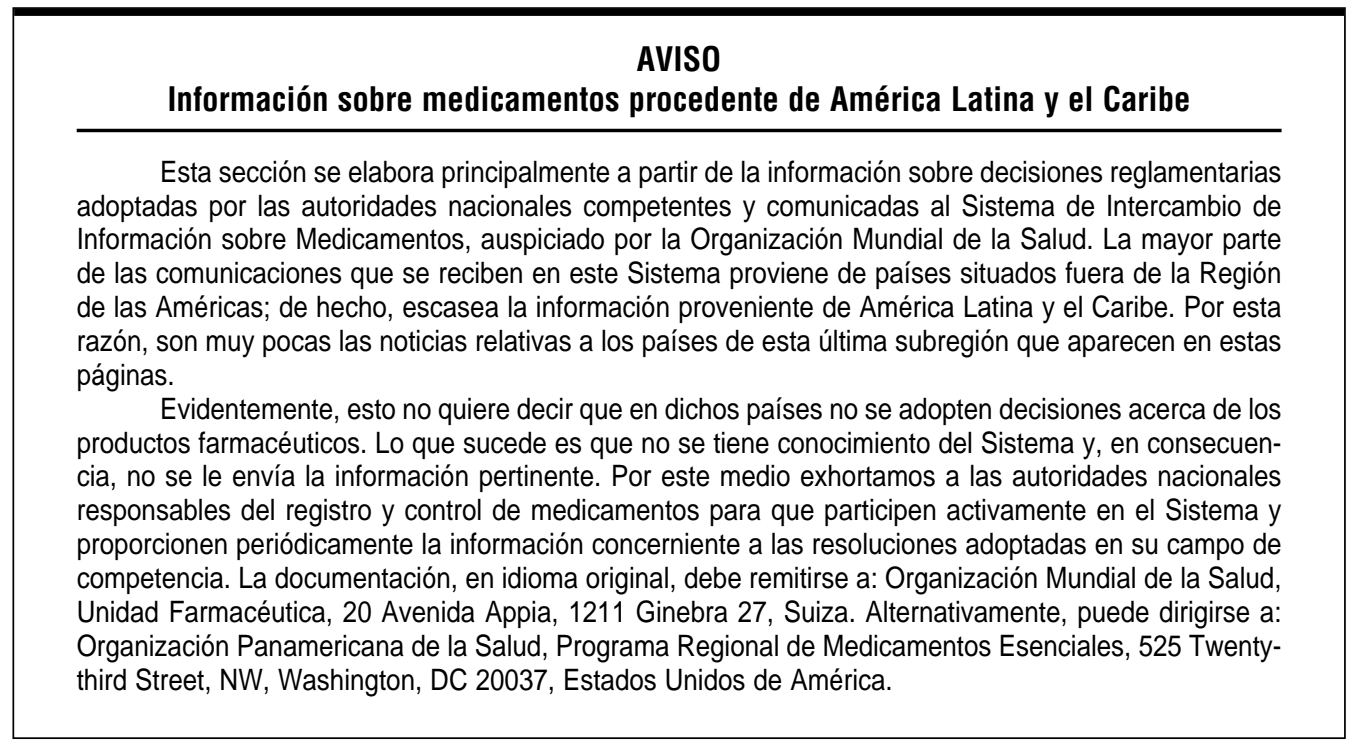

